\title{
Étude biométrique de populations d'abeilles tunisiennes
}

\author{
K Grissa 1, JM Cornuet 2*,**, K Msadda 1, J Fresnaye 2 \\ 1 Institut National Agronomique de Tunis, laboratoire de zoologie et d'apidologie, \\ 43, avenue Charles-Nicolle, Tunis, Tunisie; \\ 2 INRA, station de zoologie et d'apidologie, 84140 Montfavet, France
}

(Reçu le 25 janvier 1990; accepté le 8 mai 1990)

\begin{abstract}
Résumé - Une étude biométrique de 110 colonies a montré que la population tunisienne échantillonnée constituait un groupe homogène, sans différenciation géographique détectable. Les caractéristiques morphologiques de cette population la situent au sein de la race intermissa.
\end{abstract}

Apis mellifera intermissa / génétique des populations / biométrie / Tunisie

\section{INTRODUCTION}

L'abeille tunisienne appartient normalement à la race nord-africaine Apis mellifera intermissa, également appelée «abeille tellienne» ou encore «abeille punique». Son aire de répartition s'étend à toute l'Afrique du Nord, du Maroc à la Tunisie. Selon Ruttner (1988), les données biométriques sur cette race sont peu nombreuses. Lui-même ne dispose, semble-til, que d'une vingtaine d'échantillons pour l'ensemble des 3 pays nord-africains. Dans un travail récent, nous avons présenté des résultats plus détaillés concernant le Maroc (Cornuet et al, 1988). Dans cet article, nous étudions plus particulièrement la Tunisie en tentant de répondre aux questions suivantes:
- quelle est la structure de la population d'abeilles tunisiennes ? En particulier, peut-on discerner une différenciation en populations locales?

- quelles sont les caractéristiques biométriques de la (des) population(s) trouvée(s)?

- trouve-t-on des indices d'influence génétique de races étrangères ayant été importées en Tunisie? La Tunisie a, en effet, fait l'objet d'un certain nombre d'importations de races étrangères. Les premières datent de la colonisation française (races mellifera et ligustica). Plus récemment, dans les années 1970, un apiculteur allemand a importé un certain nombre de races (carnica, caucasica, ligustica, macedonica et mellifera). Enfin, une importation massive de 1500 colonies de Roumanie (carnica) a eu lieu en 1976.

\footnotetext{
* Correspondance et tirés à part;

** Adresse actuelle : INRA-CNRS, laboratoire de neurobiologie comparée des invertébrés, BP 23, 91440 Bures-sur-Yvette, France.
} 


\section{MATÉRIEL ET MÉTHODES}

\section{Échantillonnage}

L'objectif principal étant d'étudier les populations locales, les échantillons ont été collectés dans des ruches traditionnelles («djebehs") ou dans des ruches à cadre n'ayant jamais été transhumées. Cent dix colonies ont été échantillonnées sur l'ensemble du pays (fig 1). Toutefois, le Centre et le Sud ont fait l'objet de prélèvements plus espacés, étant donné le climat semi-aride à aride et la faible densité des colonies de ces régions. Les abeilles ont été rapportées vivantes au laboratoire où elles ont été tuées à l'acétate d'éthyle et conservées dans une solution d'alcool à $55 \%$.

\section{Caractères morphologiques et méthodes de mesure}

Les mesures ont été effectuées sur 24 ouvrières par colonie échantillonnée, les caractères retenus étant ceux décrits par Fresnaye (1981) :

- coloration : largeur de la bande jaune sur le $2^{\mathrm{e}}$ tergite abdominal;

- pilosité : longueur moyenne des poils sur le $5^{\ominus}$ tergite abdominal;

- tomentum : largeur de la bande pileuse sur le $4^{\theta}$ tergite abdominal;

- langue : longueur de la langue;

- index-A et index- $B$ : longueur des 2 nervures $A$ et $B$ de l'index cubital.

Toutes les mesures ont été effectuées au stéréomicroscope (Wild M5A) équipé d'un micromètre oculaire pour la mesure des 4 premiers caractères et d'un dispositif de Ruttner (1963) pour celle des composantes de l'index cubital.

\section{Méthodes statistiques}

La structure de la population ainsi que sa situation par rapport à des échantillons de référence ont été étudiées par analyse factorielle discriminante (Cornuet et al, 1975). La représentation des groupes dans les plans factoriels fait appel aux ellipses de confiance (Cornuet, 1982).

Les groupes individualisés par les analyses précédentes sont caractérisés par leurs statistiques élémentaires : moyennes, écarts types, histogrammes pour chacun des caractères.

\section{RÉSULTATS}

\section{Structure de l'échantillon d'abeilles tunisiennes}

Une analyse factorielle discriminante a été effectuée sur l'ensemble des abeilles tunisiennes (2620 abeilles regroupées en 110 colonies). La projection des points moyens des colonies sur les 2 plans factoriels les plus discriminants fait apparaître un nuage relativement homogène (fig 2 et 3 ). La fi-

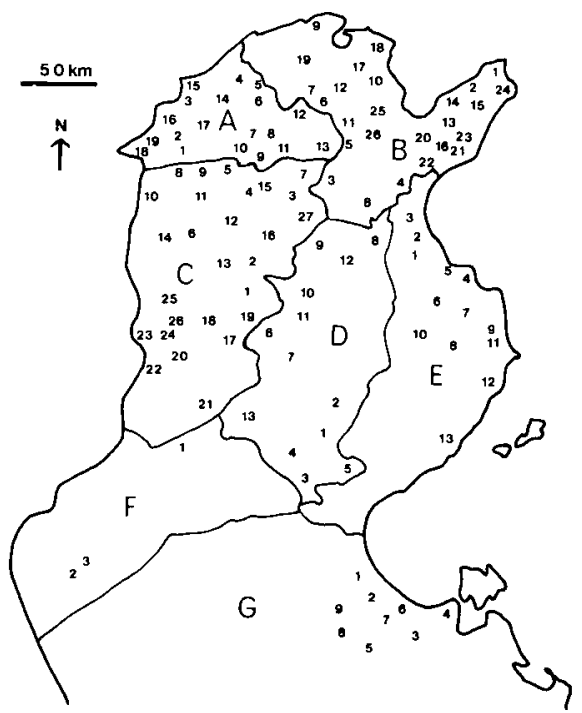

Fig 1. Répartition géographique des colonies échantillonnées. Dans les figures suivantes, les colonies seront repérées par la lettre correspondant à la région suivie du numéro porté sur la carte. 


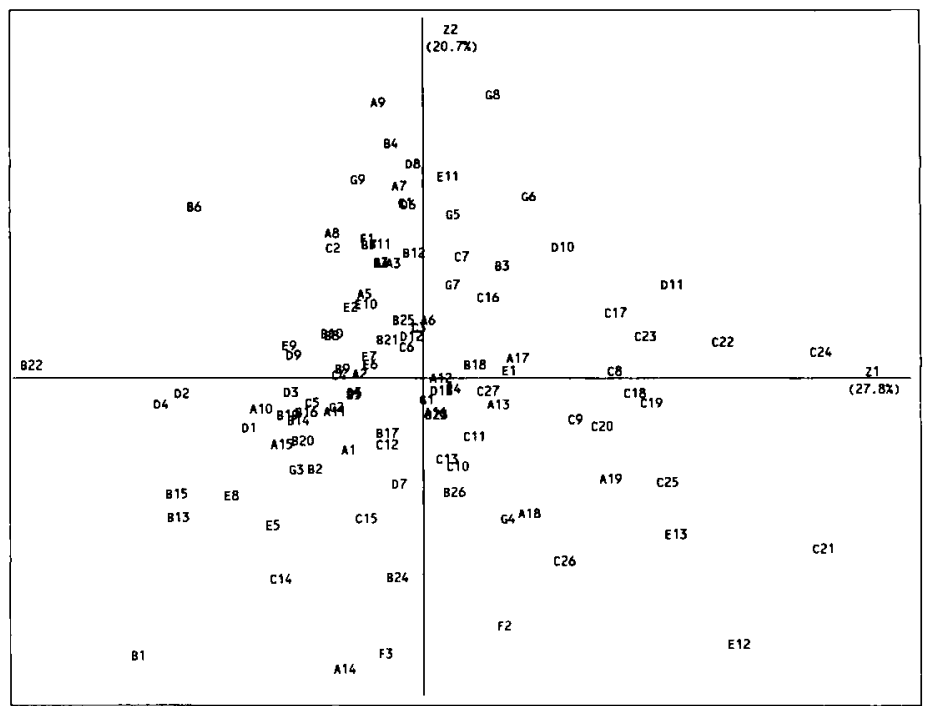

Fig 2. Analyse factorielle discriminante sur l'échantillon tunisien (2 620 abeilles regroupées en 110 colonies). Projection des points moyens des colonies sur le plan $Z_{1} Z_{2}$.

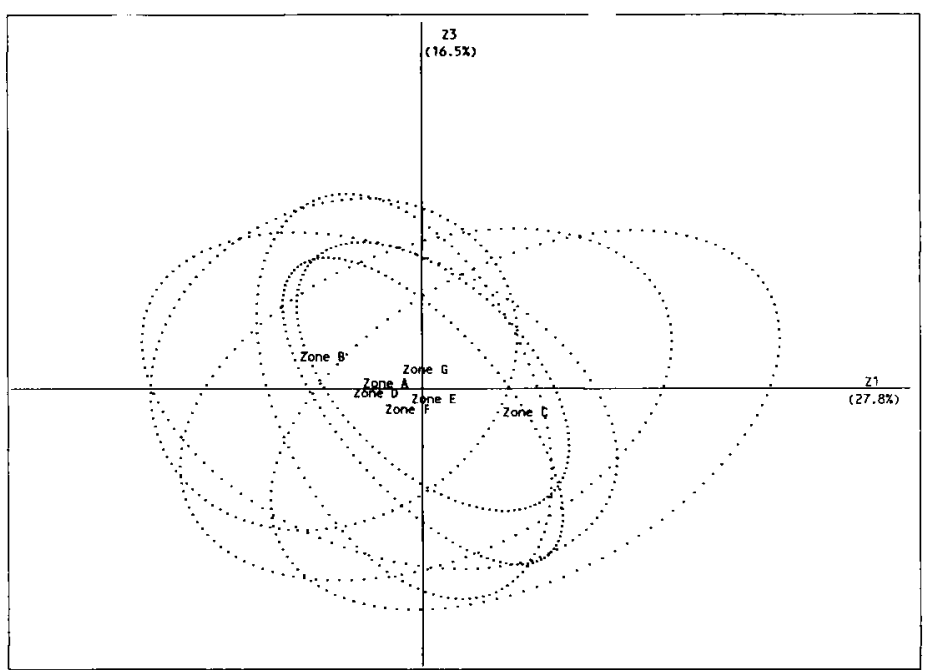

Fig 3. Analyse factorielle discriminante sur l'échantillon tunisien ( 2620 abeilles regroupées en 110 colonies). Représentation des ellipses de confiance (niveau 0,75 ) des colonies regroupées par région d'origine. 
Tableau I. Caractéristiques biométriques (en $\mathrm{mm}$ ) des abeilles tunisiennes.

\begin{tabular}{lllll}
\hline & Moyenne & Ecarts types & Mininum & Maximum \\
\hline Coloration & 0,19 & 0,059 & 0,10 & 0,30 \\
Pilosité & 0,20 & 0,017 & 0,15 & 0,24 \\
Tomentum & 0,56 & 0,057 & 0,47 & 0,75 \\
Langue & 6,40 & 0,117 & 6,03 & 6,61 \\
Index-A & 0,563 & 0,036 & 0,507 & 0,662 \\
Index-B & 0,249 & 0,021 & 0,215 & 0,270 \\
& & & & \\
\hline
\end{tabular}

gure 3 montre, en outre, qu'il ne semble pas y avoir de différenciation régionale : les ellipses de confiance (niveau 0,75 ) correspondant aux 7 régions définies sur la carte (fig 1 ) se recouvrent très largement.

Cette analyse indique donc que l'échantillon d'abeilles tunisiennes est issu d'une population morphologiquement homogène pour les 6 caractères étudiés, sans indice d'une différenciation régionale.

\section{Caractérisation morphologique de la population tunisienne}

La figure 4 représente les histogrammes des moyennes de colonies pour chacun des 6 caractères biométriques. Les distributions sont toutes unimodales.

Les moyennes générales et écarts types moyens (racine carrée du carré moyen résiduel dans l'analyse de variance comparant les moyennes de colonies) sont indiqués au tableau I. L'abeille tunisienne est donc de couleur noire $(0,19$ $\mathrm{mm})$, avec une langue de longueur moyenne $(6,4 \mathrm{~mm})$, une courte pilosité $(0,20 \mathrm{~mm})$ et un tomentum étroit $(0,56$ $\mathrm{mm})$; son index cubital moyen est de 2,26.

\section{Influence de races étrangères dans les colonies tunisiennes}

Les influences raciales se détectent sur les plans d'une analyse discriminante réalisée sur un échantillon des différentes races ayant été introduites en Tunisie, les colonies étudiées étant alors considérées comme individus supplémentaires dans l'analyse. Une position intermédiaire sur un plan discriminant entre la race présumée et une race importée est un bon indice de pollution résiduelle.

Les figures 5 et 6 montrent qu'aucune colonie n'occupe une position intermédiaire entre intermissa et l'une quelconque des 4 races ayant été importées en Tunisie (mellifera, ligustica, caucasica et carnica). La méthode utilisée ne permet donc pas de détecter d'influence génétique de l'une quelconque de ces races dans l'échantillon étudié.

\section{DISCUSSION}

La population d'abeilles tunisiennes étudiée constitue un groupe homogène, sans différenciation géographique détectable par la méthode utilisée. Plusieurs phéno- 
Coloration
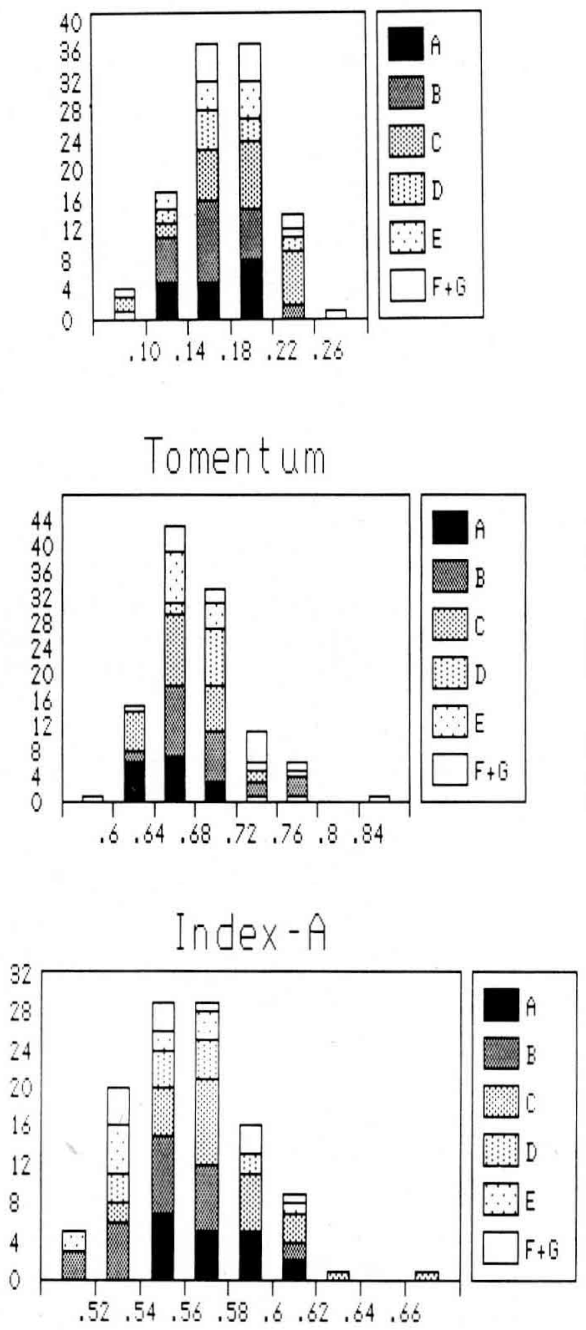

Pilosite
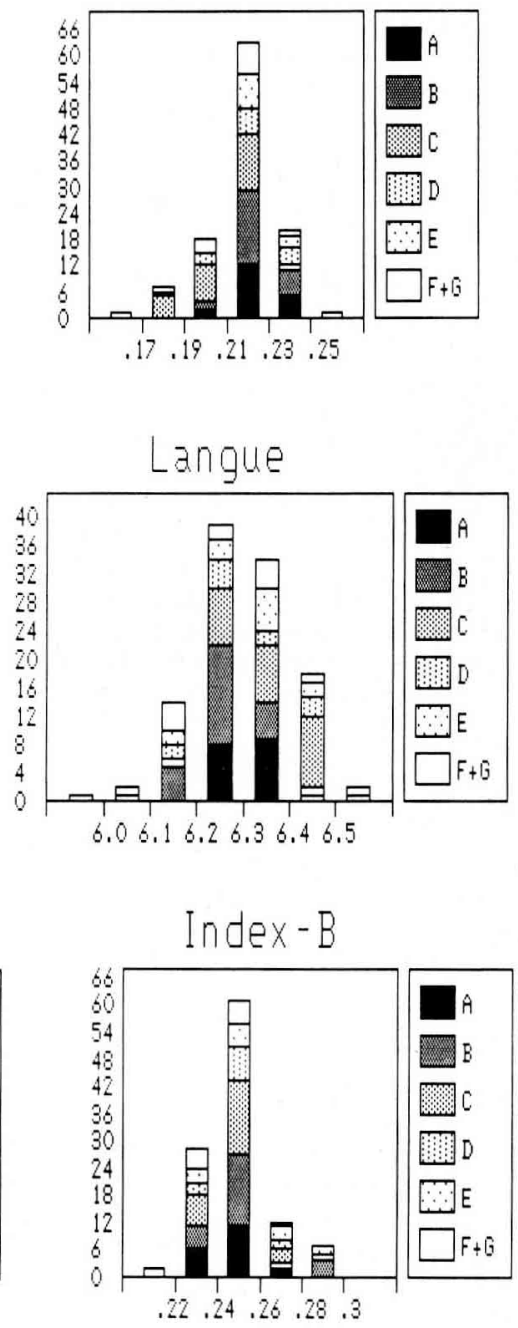

Fig 4. Histogrammes des moyennes de colonies pour les 6 caractères morphologiques étudiés. En raison de son faible effectif, la région $\mathrm{F}$ a été regroupée avec la région $\mathrm{G}$ voisine.

mènes ont pu concourir à ce résultat. D'une part, sur l'ensemble du territoire échantillonné, il n'y a pas d'obstacles naturels infranchissables susceptibles d'isoler génétiquement des groupes locaux.
D'autre part, intermissa partage avec les autres races africaines un comportement très essaimeur qui contribue à maintenir une homogénéité morphologique sur des distances importantes (Gadbin et al, 


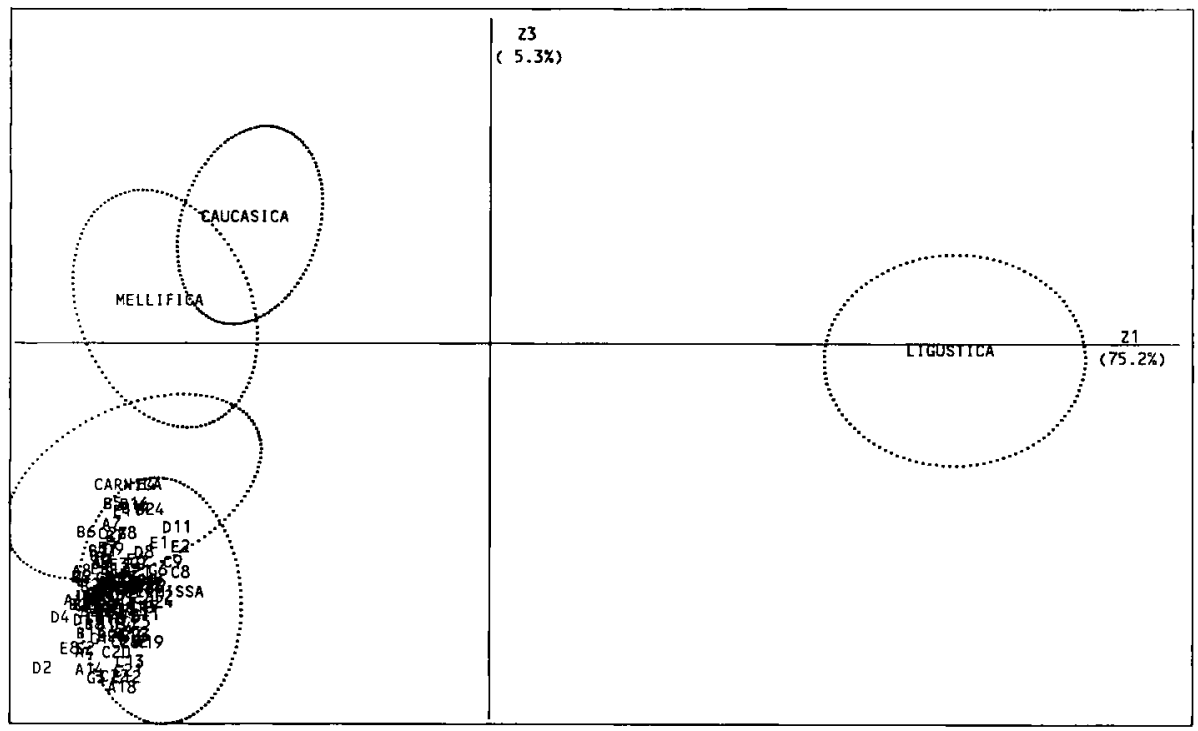

Fig 5. Analyse factorielle discriminante sur un échantillon de référence (races mellifera, ligustica, caucasica, carnica, intermissa), les colonies tunisiennes étant considérées comme individus supplémentaires dans l'analyse. Les races de références sont représentées par leur ellipse de confiance (niveau $0,95)$.

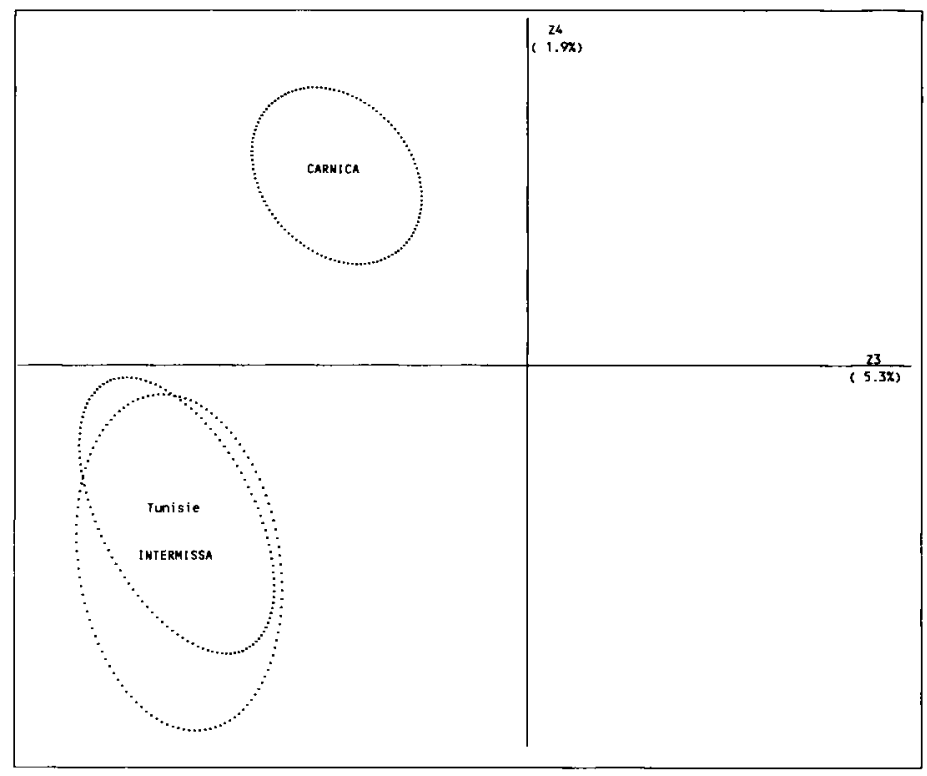

Fig 6. Analyse factorielle discriminante sur un échantillon de référence (races mellifera, ligustica, caucasica, carnica, intermissa), les colonies tunisiennes étant considérées comme individus supplémentaires dans l'analyse. Les races de références et la population tunisienne sont représentées par leur ellipse de confiance (niveau 0,95). Pour plus de clarté, seules les races de référence carnica et intermissa, mal discriminées sur la figure 5 , ont été représentées. 
1979). Enfin, une transhumance importante affecte la plupart des zones échantillonnées et contribue, par le brassage de gènes qu'elle provoque, à l'homogénéisation génétique des populations concernées.

Les figures 5 et 6 montrent l'inclusion de la population tunisienne au sein de l'échantillon de référence de la race intermissa, constitué de colonies du Maroc, d'Algérie et de Tunisie. La variabilité des colonies tunisiennes est inférieure à la variabilité de la race dans son ensemble. Les données de Ruttner (1988) concernant cette race lincitent à penser qu'une différenciation a pu s'établir entre les populations des 3 pays magrebhins. Les résultats présents vont dans le sens de cette hypothèse.

Les multiples introductions d'abeilles étrangères ne semblent pas avoir marqué d'une manière sensible la race locale. Plusieurs facteurs ont sans doute contribué à atténuer l'impact de ces introductions et à maintenir l'homogénéité de la race. D'une part, les races importées ne sont pas adaptées aux conditions climatiques particulières de la Tunisie (en particulier aux grandes chaleurs estivales). D'autre part, en raison de sa grande prolificité et de sa tendance exceptionnelle à l'essaimage, la race intermissa présente sur son territoire un avantage décisif qui lui a permis de dominer et d'absorber toutes ces abeilles introduites.

\section{Summary - Biometrical study of honey} bee populations from Tunisia. A total of 110 honey bee colonies were sampled around Tunis (fig 1). For every sampled colony, 24 workers were measured according to Fresnaye (1981) for the 6 following characters: width of the yellow strip on tergite 2 , length of hairs on tergite 5 , width of tomentum on tergite 4 , length of proboscis and wing veins $A$ and $B$ of the cubital index.

Histograms (fig 4) as well as factorial discriminant analysis show that the whole population is rather homogeneous for the 6 studied characters without any regional differentiation. This population is included in the reference Apis mellifera intermissa sample and there is no detectable genetic influence from the races that have been imported into Tunisia.

The lack of genetic differentiation can be explained by the lack of natural barriers in the country, the intense swarming behaviour of tellian bees and the rather frequent moving of hives by beekeepers. It is probable that the races imported from Europe not adapted to the ecological conditions of Tunisia have been eliminated by the better adapted, highly swarming intermissa honey bees.

\section{Apis mellifera intermissa / population genetics / biometry / Tunisia}

\section{Zusammenfassung - Biometrische Analyse der Bienenpopulationen Tune- siens. Insgesamt 110 Proben aus Bienen- völkern in Tunesien wurden gesammelt (Abb 1). Aus jedem ausgewählten Volk wurden 24 Arbeiterinnen entnommen und gemäß Fresnaye (1981) folgende sechs Merkmale an ihnen gemessen: Breite des gelben Streifens auf Tergit 2, Länge des Überhaars auf Tergit 5, Breite des Filzstrei- fens auf Tergit 4, Rüssellänge und Ader- länge $A$ und $B$ (Cubital-Index) des Flügel- geäders.}

Das Histogramm (Abb 4) zeigt ebenso wie die Faktorenanalyse, daß die ganze Population eher homogen in Bezug auf diese 6 Merkmale ist und keine regionalen 
Unterschiede auftreten. Die Population entspricht dem Referenzexemplar von Apis mellifera intermissa und es gibt keine nachweisbaren genetischen Einflüße der nach Tunesien importierten Rassen.

Das Fehlen von genetischer Differenzierung könnte mit dem Fehlen von natürlichen Barrieren im Lande, dem intensiven Schwarmverhalten der Tellbiene und der eher intensiven Wanderlust der Imker erklärt werden. Vermutlich waren die Rassen, die aus Europa importiert wurden, nicht angepaßt an die ökologischen Bedingungen von Tunesien und wurden deshalb von den besser angepaßten, leicht schwärmenden intermissa Bienen wieder verdrängt.

Apis mellifera intermissa / Populationsgenetik / Biometrie / Tunesien

\section{RÉFÉRENCES}

Cornuet JM (1982) Représentation graphique de populations multinormales par des ellipses de confiance. Apidologie 13, 15-20

Cornuet JM, Fresnaye J, Tassencount L (1975) Discrimination et classification de populations d'abeilles à partir de caractères biométriques. Apidologie 6, 147-187

Cornuet JM, Daoudi A, Mohssine EH, Fresnaye $J$ (1988) Étude biométrique de populations d'abeilles marocaines. Apidologie 19, 355366

Fresnaye J (1981) Biométrie de l'abeille, $2^{\ominus}$ éd, Echauffour (Orne), Office Inf Doc Apic, $56 \mathrm{p}$

Gadbin C, Cornuet JM, Fresnaye J (1979) Approche biométrique de la variété locale d'Apis mellifera $L$ dans le sud tchadien. Apidologie 10, 137-148

Ruttner F (1963) Die Zuchtauslese bei der Biene. NO Imkerschule W, R Neustadt, Waltergrass $6,79 \mathrm{p}$

Ruttner F (1988) Biogeography and Taxonomy of Bees. Springer-Verlag, Berlin, $284 \mathrm{p}$ 